\title{
ORIGINAL
}

\section{INCIDENCIA Y VARIABILIDAD DE LA INCAPACIDAD TEMPORAL POR TRASTORNOS DE ANSIEDAD EN LA COMUNIDAD VALENCIANA DURANTE EL 2009}

\author{
Joaquín Andani Cervera (1), Natividad Guadalajara Olmeda (2), Isabel Barrachina Martinez (2) y \\ David Vivas Consuelo (2).
}

(1) Hospital Universitario y Politécnico La Fe. Valencia. España

(2) Centro de Investigación en Economía y Gestión de la Salud. Departamento de Economía y Ciencias Sociales. Universitat Politècnica de València.Valencia. España

\begin{abstract}
RESUMEN
Fundamentos: Los trastornos de ansiedad, disociativos y somatomorfos (300) constituyen la segunda causa de incapacidad temporal (IT) en España. Esta razón justifica que el presente trabajo se centre en analizar la variabilidad en la prescripción de procesos de IT por estas patologías en los centros de salud y consultorios (CS) de la Comunidad Valenciana (CV).

Métodos: Se realizó un estudio epidemiológico poblacional transversal de variabilidad de los procesos de IT iniciados en 2009 correspondientes al 300 en los 739 CS, de los 23 departamentos de salud de la $\mathrm{CV}$, donde se prescribieron un total de 25.859 procesos de IT por 300 Se utilizaron los estadísticos de variabilidad habituales en el análisis de áreas pequeñas para determinar la variabilidad observada en la tasa de incidencia (TI) de los procesos de IT. El análisis de la varianza se utilizó para determinar el porcentaje de explicación de cada factor estudiado.

Resultados: La TI media por 100 individuos es de 1,08 con un rango de variación de 0,01 a 1,97 entre los percentiles $\mathrm{P}_{5}$ a $\mathrm{P}_{95}$. En el análisis de componentes de la varianza, el factor departamento de salud es el que explica mayor porcentaje de variabilidad $(22,12 \%)$ seguido del factor provincia $(20,21 \%)$, litoralidad $(4,65 \%)$, acreditación para la docencia $(2,44 \%)$ y tamaño poblacional asignado al CS $(2,40 \%)$.

Conclusiones: Se observa una variabilidad notable en las TI de las prescripciones de IT por 300. Los factores departamento y provincia son los que más explican esta variabilidad. Los CS con mayor presión poblacional y los acreditados tienen mayores tasas de incidencia.
\end{abstract}

Palabras clave: Ausencia por enfermedad. Trastornos mentales. Atención Primaria de Salud. Seguro por enfermedad. Administración de la Práctica médica.

Correspondencia

David Vivas

Centro de Investigación en Economía y Gestión de la Salud

Facultad de Administración y Dirección de Empresas. Edificio 7J.

Universitat Politècnica de València

Camino de Vera $\mathrm{s} / \mathrm{n}$

46022 Valencia

\section{ABSTRACT \\ Incidence and Variability of Temporary Disability Due to Anxiety Disorders during 2009 in the Valencia Community, Spain}

Background: Anxiety, dissociative and somatoform disorders (WHO-e 300) are the second cause of Temporary Disability (TD) in Spain. This is the main reason that justifies the analysis of the variability among primary health care centers (PHC) of the Valencian Community in the prescription processes of Temporary Disability for these disorders.

Methods: Epidemiological cross-sectional descriptive study of variability of TD processes initiated in 2009 corresponding to diagnosis e 300 in 739 PHC from 23 health districts in the Valencian Community, where 25,859 TD processes for the diagnosis e 300 were prescribed. Traditional indicators of variation developed for the analysis of small areas were used to determine variability in the incidence rate of TD processes. The analysis of variance was used to determine the percentage of explanation of the factors studied.

Results: The average incidence rate obtained was of 1.08 for 100 individuals. The variation range was between 0.01 and 1.97 for percentiles $\mathrm{P}_{5}$ to $\mathrm{P}_{95}$. In the variance components analysis, the factor of health district explains the highest percentage of variability (22.12), followed by the factor province $(20.21 \%)$, coastal areas $(4.65 \%)$, teaching accreditation $(2.44 \%)$ and the size of population assigned to each $\mathrm{PHC}$ $(2.40 \%)$.

Conclusions: Significant differences were observed in the incidence rate of TD processes for the diagnosis e 300. The PHCs with greater population pressure and those that are accredited had the highest rates of incidence.

Key words: Sick Leave, Mental Disorders, Primary Health Care, Disability Insurance, Medical Practice Management 


\section{INTRODUCCIÓN}

La prestación de Incapacidad Temporal (IT) es financiada por el Instituto Nacional de la Seguridad Social (INSS) en colaboración con las mutuas patronales. El presupuesto de la Seguridad Social (SS) para las prestaciones económicas por IT por contingencia común, para 2011 fue de 7.008.864.000 euros ${ }^{1}$. Su trascendencia social, económica y sanitaria es enorme, como ocurre en muchos países desarrolla$\operatorname{dos}^{2}$.

El Sistema de SS español defiende las competencias del Sistema Nacional de Salud (SNS) en el control sanitario de las altas y bajas, y faculta al INSS para establecer acuerdos de colaboración con los servicios de salud de las Comunidades Autónomas $^{3}$ y desde 2010 a los facultativos de dicho organismo para la emisión de partes de alta.

La prescripción de IT se realiza en los centros de salud (CS) por los médicos de atención primaria (AP) ${ }^{4-9}$. Un estudio ${ }^{10}$ propone que los datos de IT sean incorporados a los procedimientos de información sanitaria, y otro ${ }^{11}$ reconoce la necesidad de unificar criterios de gestión de la IT en las Unidades Médicas de Valoración de Incapacidades, para reducir la variabilidad o unificar los criterios de las prescripciones de bajas o altas. Partiendo de la hipótesis que la concesión de una baja o de un alta laboral es una prescripción médica más, es susceptible de ser analizada con instrumentos estadísticos de análisis de la variabilidad.

La variabilidad en la práctica clínica se define $^{12,13}$ como las diferencias en la prestación de servicios sanitarios entre profesionales o zonas geográficas que pueden implicar una diferente utilización de los mismos. La variabilidad en la prescripción de IT puede ser debida al paciente (edad, nivel socioeconómico, grupo étnico y sexo), al régimen del trabajador (autónomo o régimen general), al facultativo (especialidad, edad, sexo, formación y experiencia), al sistema de gestión (Mutua o INSS) o al centro asistencial (público o privado, rural o urbano, acreditación para la docencia y tamaño), entre otros factores ${ }^{14,15}$, y ha sido estudiada en relación a las intervenciones quirúrgicas ${ }^{16}$, al consumo de fármacos ${ }^{17} \mathrm{y}$ al uso de servicios clínicos ${ }^{18}$.

Entre las causas que generan situaciones de IT de mayor duración ${ }^{5,19-22}$, recurrencia ${ }^{6}$ e incapacidad permanente ${ }^{7}$, están las enfermedades mentales. En los estudios de carga de morbilidad realizados a nivel mundial, la depresión mayor unipolar figura en cuarta posición ${ }^{8}$. Según las proyecciones de la OMS para 2020 alcanzarán el 15\% del total, llegando a ser la depresión la segunda causa de morbilidad, por detrás de la cardiopatía isquémica ${ }^{9}$. Actualmente, un $20-25 \%$ de los pacientes que consultan en AP presentan síntomas relacionados con los trastornos mentales ${ }^{23-25}$.

Las enfermedades mentales se integran en el grupo 5 de los 19 que componen la Clasificación Internacional de Enfermedades, CIE-9-MC, 2010, con códigos diagnósticos desde el 290 al 319. Los trastornos de ansiedad, disociativos y somatomórfos, que se ifican con el código diagnóstico 300 (300), constituyen la segunda causa de IT en España ${ }^{26}$.

El objetivo del presente estudio es analizar la incidencia de la prescripción de procesos de IT por trastornos de ansiedad, disociativos y somatomórfos en la Comunidad Valenciana (CV) durante el año 2009, su variabilidad y los factores asociados exclusivamente con los CS causantes de dicha variabilidad, de acuerdo a la información disponible.

\section{MATERIAL Y MÉTODOS}

Diseño. Estudio epidemiológico poblacional transversal ecológico de la variabi- 
lidad de los procesos de IT por 300 en los CS de la Comunidad Valenciana.

Población y ámbito. La unidad de análisis es cada uno de los 739 CS (257 centros de salud y 482 consultorios auxiliares) de los 23 departamentos de salud de la CV. Los departamentos del 1 al 3 y parte del 4 corresponden a la provincia de Castellón, el 13 y del 15 al 22 a Alicante y el resto a Valencia. En dichos CS se prescribieron un total de 480.755 procesos de IT por todos los diagnósticos, de los cuales 25.859 correspondían al 300 , que fueron analizados en su totalidad.

Los servicios de AP son atendidos por personal sanitario: médicos de AP, médicos pediatras, enfermeros y auxiliares de enfermería, y no sanitario: auxiliares administrativos y celadores. En $10 \mathrm{CS}$ de la CV se incluyen también otros profesionales (psicólogos clínicos, odontólogos).

Fuentes de datos. Los datos de los procesos de IT se obtuvieron del Sistema de Información Ambulatoria (SIA) integrado en el sistema de información ABUCASIS de la Conselleria de Sanidad de la Generalitat Valenciana. El SIA incluye varios módulos de gestión, como la historia clínica electrónica, que registra toda la información asistencial procedente de las consultas de AP y de centros de especialidades (prescripción de procesos de IT, diagnósticos activos y pasivos, motivos de consulta, etc.).

Los datos de información poblacional se obtuvieron del Sistema de Información Poblacional (SIP) de la Agencia Valenciana de Salud, ya utilizados en trabajos anteriores ${ }^{17}$.

Medidas de resultados. El indicador de gestión sanitaria de IT es la tasa de incidencia (TI) del CS:

$$
\mathrm{TI}=(\mathrm{NP} / \mathrm{PR}) \times 100
$$

Siendo:

NP: el número de pacientes a los que se les ha prescrito un proceso de IT durante 2009.

PR: la población en riesgo o población en situación de alta o asimilada al alta en la SS, a fecha de uno de enero de 2009 , considerada constante durante el año, es decir, población entre 16 y 65 años ocupada o en situación de desempleo contributivo y susceptible de serle prescrita una baja laboral, equivalente a 2.304 .598 personas.

Se utilizó el método directo de estandarización por edad y sexo, para realizar comparaciones entre CS, considerando que todos tienen la misma distribución que la población de referencia (total de la CV). Y la estandarización indirecta para comparar cada CS con el global de la CV, considerando los estratos definidos en la tabla 1 .

Análisis estadístico. Para las comparaciones entre CS se calcularon los estadísticos de variabilidad habituales en el análisis de áreas pequeñas ${ }^{17,18,27,28}: 1$ ) razón de variación entre las áreas situadas en el percentil $5\left(\mathrm{P}_{5}\right)$ y percentil $95\left(\mathrm{P}_{95}\right)$ (cociente entre la frecuentación del área en el $\mathrm{P}_{95} \mathrm{y}$ la del área en el $\left.\mathrm{P}_{5}, \mathrm{RV}_{5-95}\right), 2$ ) razón de variación $\mathrm{RV}_{25-75}, 3$ ) coeficiente de variación (cociente entre la desviación estándar y la media, calculado sin las áreas por debajo del $\mathrm{P}_{5}$ o por encima del $\left.\left.\mathrm{P}_{95}\right)\left(\mathrm{CV}_{5-95}\right), 4\right)$ coeficiente de variación ponderado por el número de registros de cada área $\left(\mathrm{CVW}_{5}\right.$ ${ }_{95}$ ). Este coeficiente está ponderado por tamaño poblacional de cada CS y soporta mejor que el coeficiente de variación la presencia de tamaños poblacionales diferentes en los CS y 5) componente sistemático de la variación $\left(\mathrm{CSV}_{5-95}\right)$, a mayor CSV mayor variación sistemática y no debida al $\operatorname{azar}^{28}$.

Para comparar cada CS con el global de la $\mathrm{CV}$, se calculó la Razón de Incidencia 
Tabla 1

Distribución de la población laboral de la Comunidad Valenciana por estratos (grupos de edad y sexo) y TI

\begin{tabular}{|c|c|c|c|c|}
\hline \multicolumn{2}{|c|}{ Mujeres (1.182.705) } & Población laboral & \multicolumn{2}{c|}{ Hombres (1.212.893) } \\
\hline TI & $\%$ & Grupos de edad & $\%$ & TI \\
\hline 0,649128 & $14,97 \%$ & $16-24$ & $14,91 \%$ & 0,27584 \\
\hline 1,558598 & $25,02 \%$ & $25-34$ & $25,76 \%$ & 0,8511 \\
\hline 1,575544 & $24,75 \%$ & $35-44$ & $25,89 \%$ & 0,958521 \\
\hline 1,476487 & $20,69 \%$ & $45-54$ & $20,22 \%$ & 0,950091 \\
\hline 1,099822 & $14,57 \%$ & $55-64$ & $13,22 \%$ & 0,922607 \\
\hline
\end{tabular}

Estandarizada (RIE) como cociente entre eventos observados y esperados. Los eventos esperados de cada CS se obtuvieron del producto de la TI de la CV para cada estrato por la población de cada estrato en el CS. Se estudiaron los CS con RIE inferior o superior en más de un $50 \%$ a la RIE del conjunto de la CV, cuyo valor es 1 .

Con el análisis de componentes de la varianza se compara el porcentaje de variabilidad con que contribuyen cada uno de los factores: departamento de salud, provincia, litoral marítimo del municipio, tamaño poblacional asignado al CS y acreditación docente de formación sanitaria especializada. La figura de medias con la representación de los intervalos LSD (Least Significant Difference) de Fisher permite visualizar e interpretar las diferencias significativas para los factores considerados.

\section{RESULTADOS}

Las enfermedades mentales motivaron $30.496(7,53 \%)$ procesos de IT iniciados en la CV durante el 2009. El 300 supuso
$26.069(63,68 \%)$ diagnósticos incluidos en el grupo de enfermedades mentales y el $5,38 \%$ de todos los diagnósticos. Las enfermedades mentales causaron $3.965 .902(14,98 \%)$ días de ausencia al trabajo, por detrás de las enfermedades del sistema osteomuscular $(29,83 \%)$ que son las que más días de baja causan.

La TI por 300 en las mujeres es $1,34 \mathrm{y}$ en los hombres 0,82 siendo las diferencias mayores en el intervalo de 25-34 años y menores en el grupo de 55-64. El grupo de edad con mayor TI en ambos sexos es el de 35-44 años (tabla 1).

Por cada 100 individuos incluidos en la PR de los 739 CS se prescribieron, en promedio, 1,0794 procesos de IT por 300 (tabla 2).

Los valores de las tasas estandarizadas excluyendo el $10 \%$ ( $5 \%$ por cada lado) de CS con valores extremos, indican que el rango de variación va de 0,01 en el $\mathrm{P}_{5}$ a 1,951 en el $\mathrm{P}_{95}$, lo que significa que la PR del CS en el $\mathrm{P}_{95}$ recibió 195 veces más procesos de IT por 300 que la del CS en el $\mathrm{P}_{5}\left(\mathrm{RV}_{5-95}=195,052\right)$. Igualmente, la PR 


\section{Tabla 2}

\section{Análisis estadístico de la variabilidad de los procesos de Incapacidad Temporal por código 300 en los CS}

\begin{tabular}{|c|c|c|}
\hline \multirow{2}{*}{ Datos crudos } & Casos (Procesos de Incapacidad Temporal iniciados) * & 25.859 \\
\hline & Tasa de incidencia media * & 1,0794 \\
\hline \multirow{7}{*}{$\begin{array}{l}\text { Tasa de incidencia } \\
\text { estandarizada por } \\
\text { método directo }\end{array}$} & Tasa de incidencia mínima * & 0 \\
\hline & Tasa de incidencia máxima * & 10,214 \\
\hline & Percentil5 * & 0,01 \\
\hline & Percentil25* & 0,28 \\
\hline & Percentil50* & 0,796 \\
\hline & Percentil75* & 1,233 \\
\hline & Percentil95* & 1,951 \\
\hline \multirow{6}{*}{$\begin{array}{c}\text { Estadísticos de } \\
\text { Variabilidad }\end{array}$} & Razón de Variación 5-95 ** & 195,052 \\
\hline & Razón de Variación 25-75 *** & 4,4 \\
\hline & Coeficiente de Variación 5-95** & 0,45 \\
\hline & Coeficiente de Variación Ponderada 5-95** & 0,323 \\
\hline & Coeficiente Sistemático de Variación * & 0,157 \\
\hline & Coeficiente Sistemático de Variación 5-95** & 0,056 \\
\hline \multirow{3}{*}{$\begin{array}{l}\text { Tasa de Incidencia } \\
\text { estandarizada por } \\
\text { método indirecto }\end{array}$} & CS con Tasa de Incidencia $<50 \%$ del conjunto de población* & $72(9,74 \%)$ \\
\hline & CS con Tasa de Incidencia $>50 \%$ del conjunto de población * & $115(15,56 \%)$ \\
\hline & CS con Tasa de Incidencia $\diamond 50 \%$ del conjunto de población $*$ & $187(25,30)$ \\
\hline
\end{tabular}

- En 739 centros de salud y consultorios auxiliares (CS), ** En los CS incluidos entre los percentiles 5 y 95 , *** En los CS incluidos entre los percentiles 25 y 75 .

del CS en el $\mathrm{P}_{75}$ recibió 4,4 veces más procesos de IT que la del CS en el $\mathrm{P}_{25}\left(\mathrm{RV}_{25-}\right.$ $\left.{ }_{75}=4,400\right)$, lo que da una idea de la variabilidad en el $50 \%$ de las TI acumulada.

El $\mathrm{CV}_{5-95}$ tuvo un valor de 0,450; el $\mathrm{CVW}_{5-95}$ de 0,323 y el CSV para el conjunto de las tasas estandarizadas y para aquellas incluidas entre los percentiles $5 \mathrm{y}$ $95\left(\mathrm{CSV}_{5-95}\right)$, del 0,157 y 0,056, respectivamente.

Los CS con TI estandarizada inferior en más de un $50 \%$ a la del conjunto de la $\mathrm{CV}$ ( $\mathrm{TI}=1,0794)$, o lo que es lo mismo, con una RIE menor que 0,5 fueron 72
$(9,74 \%)$, mientras que aquellos con una TI estandarizada superior en más de un $50 \%$ fueron $115(15,56 \%)$, la mayoría de ellos ubicados en la capital de Valencia y municipios de alrededor.

En el análisis de componentes de la varianza (tabla 3 ) se excluyeron aquellos CS cuya TI estandarizada fue nula, y otro CS con valor 10 por ser 5 veces mayor que el siguiente menor.

El factor departamento de salud es el que explicó mayor porcentaje de variabilidad $(22,12 \%)$ seguido del factor provincia $(20,21 \%)$, litoralidad $(4,65 \%)$, acredi- 
Tabla 3

Análisis de Componentes de la varianza para la Tasa de Incidencia con los siguientes factores fijos: departamento de salud, provincia, litoralidad, acreditación docente y tamaño poblacional

\begin{tabular}{|l|c|c|c|c|c|}
\hline Fuente & Suma de cuadrados & $\mathrm{gl}$ & Cuadrado medio & Comp.Var. & $\%$ \\
\hline Total (corregido) & 42,9595 & 574 & & & \\
\hline Departamento de salud & 10,4434 & 22 & 0,474699 & 0,0167328 & $\mathbf{2 2 , 1 2}$ \\
\hline Residual & 32,5161 & 552 & 0,058906 & 0,058906 & 77,88 \\
\hline Total (corregido) & 183,699 & 574 & & & \\
\hline Provincia & 23,6082 & 2 & 11,8041 & 0,0709111 & 20,21 \\
\hline Residual & 160,091 & 572 & 0,27988 & 0,27988 & 79,79 \\
\hline Total (corregido) & 42,9595 & 574 & & & 0,00357621 \\
\hline Litoralidad & 0,970753 & 1 & 0,970753 & 4,65 \\
\hline Residual & 41,9887 & 573 & 0,0732787 & 0,0732787 & 95,35 \\
\hline Total (corregido) & 42,9595 & 574 & & & \\
\hline Acreditación docente & 0,505675 & 1 & 0,505675 & 0,00185455 & 2,44 \\
\hline Residual & 42,4538 & 573 & 0,0740904 & 0,0740904 & 97,56 \\
\hline Total (corregido) & 42,9595 & 574 & & & \\
\hline
\end{tabular}

Tabla 4

Análisis de Componentes de la Varianza para la Tasa de Incidencia con los factores: provincia y departamento de salud

\begin{tabular}{|c|c|c|c|c|c|}
\hline Fuente & Suma de Cuadrados & Gl & Cuadrado Medio & Comp Var.. & $\%$ \\
\hline Total (corregido) & 183,699 & 574 & & & \\
\hline Provincia & 23,6082 & 2 & 11,8041 & 0,0666505 & 19,03 \\
\hline Departamento & 183,699 & 20 & 0,947119 & 0,0279168 & 7,97 \\
\hline Error & 23,6082 & 552 & 0,255704 & 0,255704 & 73,00 \\
\hline
\end{tabular}

tación para la docencia $(2,44 \%)$ y tamaño poblacional asignado al CS $(2,40 \%)$. Los gráficos LSD (figura 1) muestran diferencias estadísticamente significativas entre los CS de Valencia frente a los de Castellón y Alicante, del litoral y del interior, y entre los CS acreditados o no para la docencia, en los que la TI es mucho mayor. Igualmente existen diferencias estadísticamente significativas entre los CS con una población asignada mayor de 20.000 personas, y los de menos de 2.000 personas.

También se realizó el análisis conjuntamente para los factores provincia $(19,03 \%)$ y departamento de salud (anidado en provincia) $(7,97 \%)$. (tabla 4$)$. 
Figura 1

Gráficos LSD de los factores departamento, provincia, litoralidad, acreditación y tamaño de la población

Medias y $95,0 \%$ Intervalos LSD

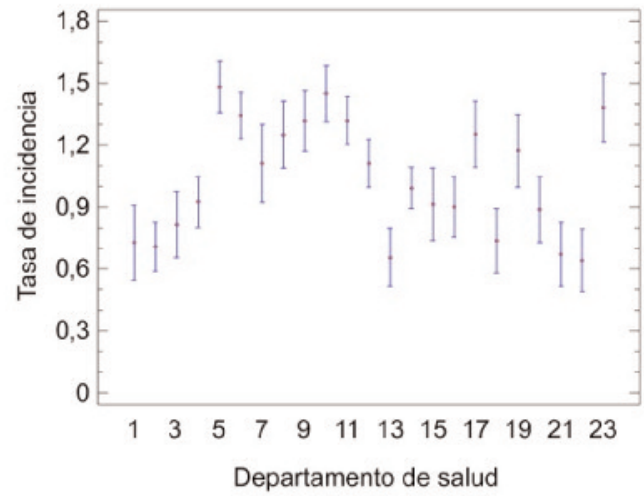

Los departamentos 1 al 3 y parte del 4 corresponden a la provincia de Castellón, el 13 y del 15 al 22 a Alicante, y el resto a Valencia

Medias y $95,0 \%$ Intervalos LSD

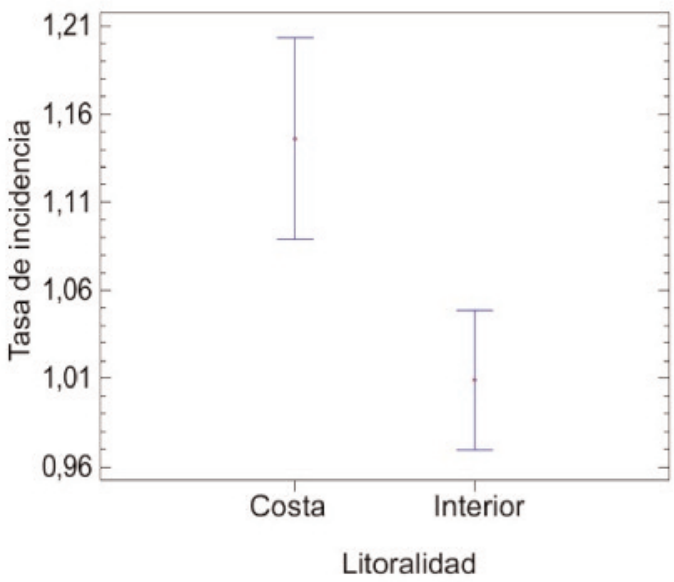

Medias y $95,0 \%$ Intervalos LSD
Medias y $95,0 \%$ Intervalos LSD

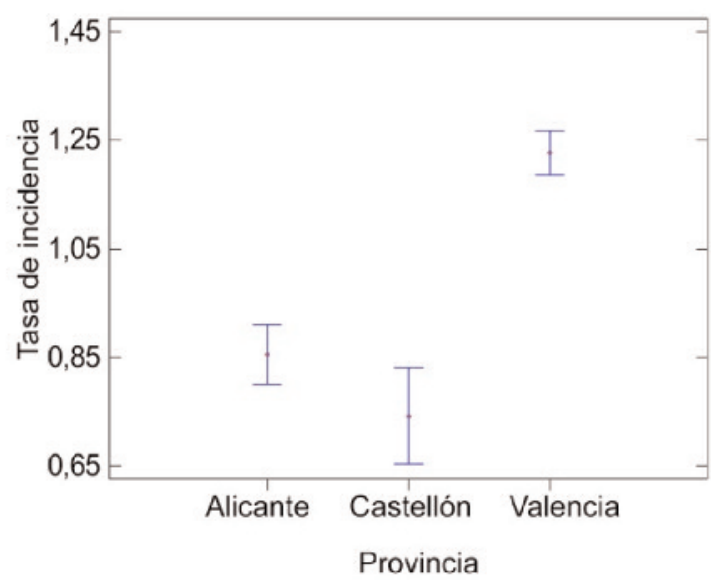

Medias y $95,0 \%$ Intervalos LSD

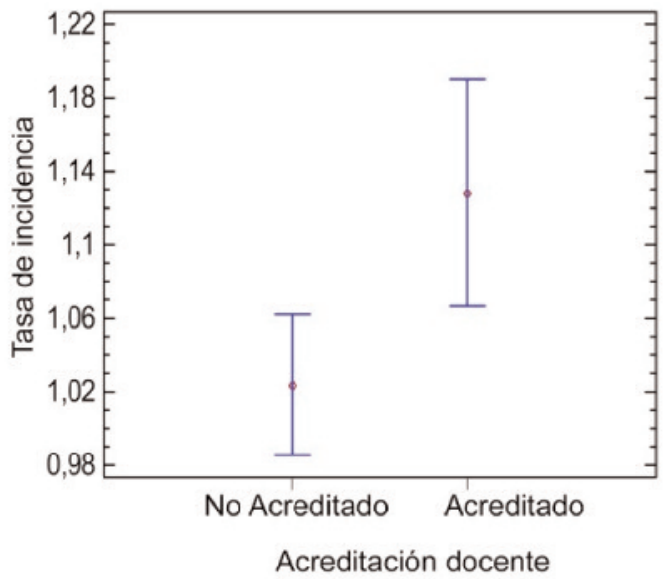

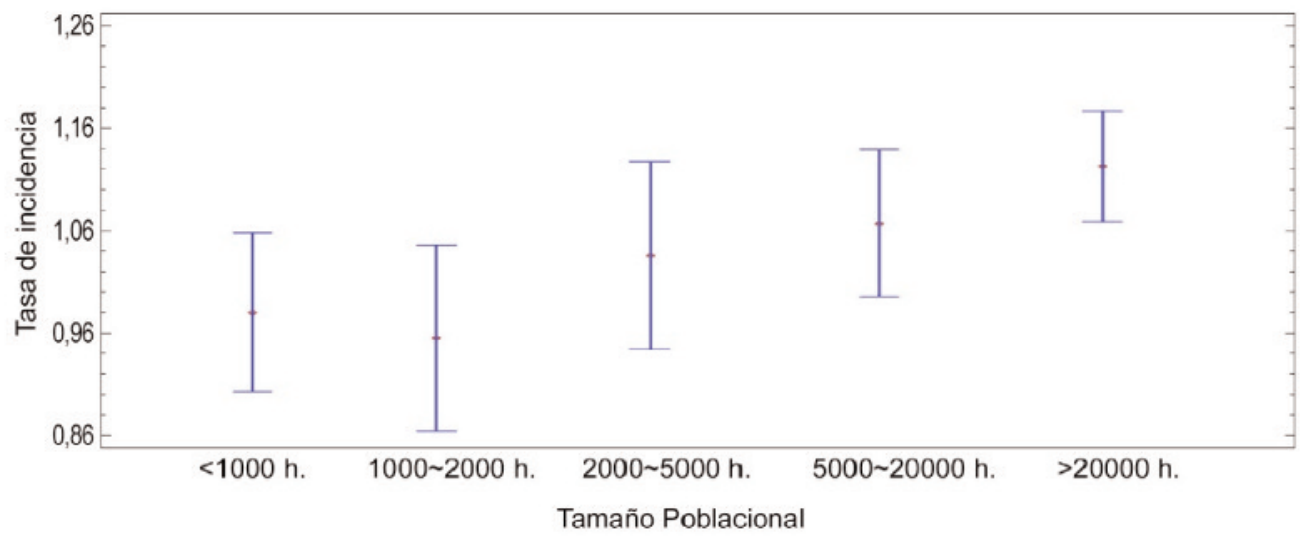




\section{DISCUSIÓN}

El factor de los CS más influyente en la variabilidad es el departamento de salud, resultado de gran relevancia por ser clave en la gestión sanitaria. Le sigue el factor provincia en la que se ubica el CS. La mayoría de los CS con TI por 300 superior en más de un $50 \%$ a la TI media están situados en la provincia de Valencia, sobre todo en la capital y los municipios próximos.

Los restantes factores (litoralidad, acreditación docente y tamaño poblacional asignado al CS), aunque en menor grado, también son estadísticamente significativos y merecen ser tenidos en consideración para la mejora de la gestión sanitaria. Los CS del litoral tienen una TI mayor que los del interior y los que tienen una población asignada de más de 20.000 personas que los de menos de 2.000. Teniendo en cuenta que los CS con mayor población asignada están ubicados en las áreas más pobladas (el litoral y la capital de Valencia) se deduce que la mayor densidad de población da lugar a una mayor TI. Sus causas son desconocidas y deberían ser objeto de estudios posteriores.

El hecho de que los CS acreditados tengan una mayor TI que los no acreditados puede interpretarse como que un mayor conocimiento de los médicos sobre los trastornos de ansiedad, da lugar a una mayor prescripción de IT por 300. Por tanto, la formación puede ser considerada como un factor de variabilidad en la prescripción entre los profesionales, tal como se ha encontrado en otro trabajo ${ }^{10}$, aunque su bajo nivel de significación estadística nos obliga a aceptar este dato con cierta cautela. De ninguna manera puede afirmarse que la variabilidad se deba a una actuación inapropiada de los profesionales o a un problema de calidad en las actuaciones médicas.
Los resultados de incidencia en las enfermedades mentales y su relación con la IT $(7,53 \%$ de los procesos de IT iniciados y $14,98 \%$ de los días de ausencia al trabajo por enfermedad) son coincidentes con otros estudios ${ }^{5-7,19-22}$.

Se demuestra una gran influencia del sexo. Las mujeres causan más baja por IT coincidiendo con unos estudios ${ }^{7,15,21,22}$ y al contrario que otros ${ }^{5,6,19-21}$, ya que esta diferencia depende de diversas circunstancias como la propia causa de la incapacidad ${ }^{19}$. También la edad da lugar a diferentes TI. En nuestro estudio, los grupos de menor y mayor edad obtienen menor TI, como en otros trabajos ${ }^{19-21}$. Estos resultados confirman la necesidad de aislar el efecto sexo y edad, estandarizando a la población para comparar entre los CS y con el conjunto de la $\mathrm{CV}$, tal y como proceden otros auto$\operatorname{res}^{14,17,18}$.

De la comparación con otros estudios de variabilidad realizados en otros ámbitos de la salud, se puede considerar la prescripción de procesos de IT por 300 en la CV, de variabilidad notable y de importante trascendencia en la gestión. Así se definió la fractura de cadera ${ }^{16}$ como un proceso de baja variabilidad, situando su $\mathrm{RV}_{5-95}$ en 2,39, su $\mathrm{RV}_{25-75}$ en 1,49, su $\mathrm{CV}_{5-95}$ en 0,22 y su $\mathrm{CSV}_{5-95}$ en 0,04 . También se refirió al consumo de antihipertensivos ${ }^{17}$ de notable variabilidad con los siguientes resultados $\mathrm{RV}_{5-95}=1,35-3,99 ; \mathrm{RV}_{25-75}=1,24-1,54$, $\mathrm{CV}_{5-95}=0,13-0,31$ y su CSV $\mathrm{CS}_{5-95}=0,02-0,07$. Se consideró la variabilidad en la utilización de los servicios de urgencias hospitalarias ${ }^{18}$ como de trascendental con los estadísticos $\mathrm{RV}_{5-95}=2,49-3,66 ; \mathrm{RV}_{25-75}=1,37$ 1,59 y CV $_{5-95}=0,32-0,43$.

Los resultados aconsejan la utilización de guías de práctica clínica en salud mental, eliminando las posibles barreras ${ }^{29}$ que puedan impedir su empleo, mejorar la atención al paciente y disminuir la variabilidad médica, garantizando criterios equi- 
tativos en la concesión de bajas laborales por IT.

No se han estudiado los procesos de IT de pacientes incluidos en los mutualismos administrativos (MUFACE, MUGEJU e ISFAS) $(2,73 \%$ de la PR) cuyos partes de baja, confirmación y alta no se extienden en los modelos oficiales del SNS.

La variabilidad debida a características individuales de los pacientes, excepto para la edad y el sexo, tampoco pudo ser analizada por ser la unidad de análisis los CS.

Como limitación principal del estudio hay que reseñar que no se dispone de información de repeticiones de IT en una misma persona. Además, el SIA de ABUCASIS permite la extracción de todos los procesos de IT por 300 , pero no la de todos los pacientes con ese mismo diagnóstico, por lo que no pudo compararse las diferencias entre pacientes con proceso de IT respecto del resto mediante el CMBD.

En conclusión, a pesar de las limitaciones señaladas, nuestro trabajo corrobora la existencia de variabilidad en los criterios de prescripción de bajas y altas por IT en los procesos estudiados y caracteriza algunas de las causas. No obstante se requieren investigaciones adicionales para tipificar mejor la variabilidad en la prescripción de baja por IT en esta y en otras enfermedades. Con este trabajo se abre una línea de investigación donde se incorporen otras variables y enfoques.

\section{AGRADECIMIENTOS}

Se agradece especialmente la colaboración de Laura B Mora Robles, Germana Llatas Serrano y Reme Gómez Almodóvar.

\section{BIBLIOGRAFÍA}

1. Seguridad Social. Estadísticas e informes [citado 11 de mayo 2011]. Disponible en:

http://www.seg-social.es/ Internet_1/Estadistica/Est/Presupuesto_aprobado/Gastos/index.htm
2. Roelen CAM, Koopmans PC, Anema JR, et al. Recurrence of medically certified sickness absence according to diagnosis: A sickness absence register study. J Occup Rehabil. 2010; 20:113-21.

3. Andani J. Variabilidad en la práctica médica de prescripción de procesos de Incapacidad Temporal, por código diagnóstico 300 de la CIE, en la Comunidad Valenciana [Tesis doctoral]. Valencia: Universitat Politècnica de València; 2010.

4. Castejón J, Benavides FG, Gimeno D, et al. Calidad del diagnóstico médico en la certificación de la Incapacidad Temporal por enfermedad común. Aten Primaria. 2006; 37:142-7.

5. Koopmans PC, Roelen CAM, Bültmann U, et al. Gender and age differences in the recurrence of sickness due to common mental disorders: a longitudinal estudy.BMC Public Health. 2010; 10:426.

6. Aurrekoetxea Agirre JJ, Sanzo Ollakarizketa JM, Zubero Oleagoitia E, et al. Repetición de procesos de incapacidad temporal según diagnóstico. Aten Primaria. 2009; 41:439-45.

7. López Cuenca S, Albaladejo Vicente R, Villanueva Orbáiz R, et al. Análisis de la Incapacidad Temporal en trabajadores de la rama sanitaria de un área de salud. Aten Primaria. 2006; 38:550-4.

8. Ayuso Mateos JL. Depresión: Una prioridad en salud pública. Med Clin (Barc). 2004; 123:181-6.

9. Murray CJL, López AD. Alternative projections of mortality and disability by cause, 1990-2200: Global Burden of Disease Study. Lancet. 1997; 349:1498-504.

10. Benavides FG. Utilidad sanitaria de la Incapacidad Temporal. Aten Primaria. 2005; 36:388-9.

11. Álvarez Theurer E, Vaquero Abellán M. Mejora de la calidad de una unidad médica de valoración de incapacidad laboral. Med Segur Trab. 2007; 3: 21-34.

12. McPherson K. Cómo debería modificarse la política sanitaria ante la evidencia de variaciones en la práctica médica. Variaciones en la Práctica Médica. 1995; 7:9-17.

13. Marión Buen J, Peiró S, Márquez Calderón S, et al. Variaciones en la práctica médica: importancia, causas e implicaciones. Med Clin (Barc).1998; 110:382-90.

14. Molinos Castro S, González Martínez R, Cimas JE, et al. Variabilidad de la práctica médica ante una sinusitis en función del consumo de recursos. Aten Primaria. $2000 ; 26: 23-37$ 
15. Benavides FG, Torá I, Martínez JM, et al. Evaluación de la gestión de los casos de incapacidad temporal por contingencia común de más de 15 días en Cataluña. Gac Sanit. 2010; 24:215-19.

16. Ruiz Jareño L. Variaciones geográficas en la cirugía de la patología degenerativa de raquis en el Sistema Nacional de Salud [tesis doctoral]. Valencia: Universitat de València; 2009.

17. Sanfélix-Gimeno G, Peiró S, Librero J. Variabilidad en la utilización de antihipertensivos entre las zonas básicas de salud de la Comunidad Valenciana. Gac Sanit. 2010; 24:397-403.

18. Peiró S, Librero J, Ridao M, et al. Variabilidad en la utilización de los servicios de urgencias hospitalarios del Sistema Nacional de Salud. Gac Sanit. 2010; 24:6-12.

19. García-Díaz AM, Pértega-Díaz S, Pita-Fernández $\mathrm{S}$, et al. Incapacidad Temporal: características en un centro de salud durante el período 2000-2002. Aten Primaria. 2006; 37:22-9.

20. González-Barcala FJ, Cadarso-Suárez C, ValdésCuadrado L, et al. Determinantes de la duración de la Incapacidad Temporal y la vuelta al trabajo en un área sanitaria de Galicia. Aten Primaria. 2006; 37:431-8.

21. Álvarez Theurer E, Llergo Muñoz A, Vaquero Abellán M. Análisis de la duración de los periodos de incapacidad temporal por procesos en Andalucía. Factores asociados. Aten Primaria. 2009;41:387-93.

22. Roelen CAM, Koopmans PC, Hoedeman R, et al. Trends in the incidence of sickness absence due to common mental disorders between 2001 and 2007 in the Netherlands. Eur J Public Health. 2009; 19:625630

23. Ansseau M, Dierick M, Buntinkx F, et al. High prevalence of mental disorders in primary care. $\mathrm{J}$ Affect Disord. 2004; 78:49-55

24. López-Bastida J, Serrano-Aguilar P, Duque-González B. Costes socioeconómicos de las enfermedades mentales en las islas Canarias en 2002. Aten Primaria. 2004; 34:32-8.

25. Sicras Mainar A, Rejas Gutierrez J, Navarro Artieda R, et al. Costes y patrón de uso de servicios en pacientes que demandan atención por problemas mentales en asistencia primaria. Gac Sanit. 2007; 21:306-13.

26. Instituto Nacional de la Seguridad Social [sede web]. Madrid: Ministerio de Trabajo y Inmigración. [acceso 14 de junio de 2010]. Estadísticas e Informes Incapacidad Temporal. Ejercicio 2009. Disponible en: http://www.seg-social.es/Internet_1/Estadistica/Est/Otras Prestaciones de la Seguridad Social/ Incapacidad_Temporal/Ejercicio_2009/index.htm

27. Librero J, Rivas F, Peiró S, et al. Metodología del Atlas de variaciones en cirugía ortopédica y traumatología en el Sistema Nacional de Salud. Atlas Var Pract Med Sist Nac Salud. 2005; 1: 43-48.

28. Ibáñez B, Librero J, Bernal-Delgado E, et al. Is there much variation in variation? Revisiting statistics of small area variation in health services research. BMC Health Services Research. 2009; 9:60. Disponible en: http://www.biomedcentral.com/14726963/9/60.

29. Cabana MD, Rand CS, Powe NR, et al. Why don't physicians follow clinical practice guidelines? JAMA. 1999; 15: 1458-1467. 